\title{
Effectiveness of Kangaroo Mother Care by Role- Playing Method on Mother's Resilience and Breastfeeding Self-Efficacy in Neonatal Intensive Care Unit, a Randomized Controlled Trial
}

fateme mohammadi

Hamadan University of Medical Sciences School of Nursing and Midwifery

\section{Behnaz Basiri}

Hamadan University of Medical Sciences Medical School

\section{Majid Barati}

Hamadan University of Medical Sciences School of Public Health

\section{Salman Khazaei}

Hamadan University of Medical Sciences

\section{Seyedeh Zahra Masoumi}

Hamadan University of Medical Sciences School of Nursing and Midwifery

\section{Efat Sadeghian}

Hamadan University of Medical Sciences School of Nursing and Midwifery

\section{Mark Gillespie}

West of Scotland Centre for Postgraduate Dental Education: Glasgow Dental Education Centre

Khodayar Oshvandi ( $\nabla$ saraghaderi6392@yahoo.com )

Mother and Child Care Research Center, Hamadan University of Medical Sciences

\section{Research}

Keywords: kangaroo mother care, role-playing, resilience, breastfeeding self-efficacy, neonatal intensive care unit

Posted Date: May 7th, 2021

DOI: https://doi.org/10.21203/rs.3.rs-453688/v1

License: (9) (1) This work is licensed under a Creative Commons Attribution 4.0 International License. Read Full License 


\section{Abstract \\ Background}

The purpose of the study is to evaluate the effectiveness of kangaroo mother care by role-playing method on mother's resilience and breastfeeding self-efficacy in neonatal intensive care unit.

\section{Methods}

In this one-blind a randomized controlled trial,78 mothers of infants admitted to neonatal intensive care unit were randomly assigned into two groups: role-playing method $(n=39)$, routine method $(n=39)$. The mothers of the intervention group were trained individually in two 30-minute sessions. After training, mothers performed kangaroo mother Care for their infants three times a day and each time 30 min for 7 days. Mothers in the control group received routine embrace care training (booklet training). Questionnaires were collected before the intervention, one day after and one week after intervention. Data analyses were conducted using SPSS version 22.

\section{Results}

mother's resilience and breastfeeding self-efficacy in the study groups was significant at the end of the two among groups. Funding demonstrated a statistically significant difference in the score of the resilience $(P=<0.001)$ and breastfeeding self-efficacy $(P=<0.001)$ in the each group one day and one week after performing the intervention. In addition, revealed a statistically significant difference between the both groups in of resilience score $(P<0.01)$ and breastfeeding self-efficacy $(P<0.01)$ one day and one week after performing the intervention $(P<0.01)$, So that the role playing method was more effective than the routine method (booklet training).

\section{Conclusion}

Training kangaroo mother care by role-playing and routine methods were both effective in mother's resilience and breastfeeding self-efficacy, but role-playing method was More effective and can be recommended role playing and routine methods as therapeutic care methods in clinical settings at the beginning of the admission of infants in the neonatal intensive care unit to improve mother's resilience and breastfeeding self-efficacy.

\section{Background}

The neonatal period considers as the most critical and essential period in terms of growth and development [1], because newborns experience unstable conditions in the first hours and days of birth which puts them in the challenge of adapting themselves to extra uterine life. Meanwhile, preterm infants 
show less ability to adapt to the world around them [1,2]. Consequently, many preterm infants may admit to the neonatal intensive care unit because of respiratory problems, temperature instability, blood pressure, heart rate, respiratory and heart problems, and neurological diseases at the very beginning days of their life [3]. Epidemiological studies reveal that annually 9.6 to 12.9 million preterm babies are born, which accounts for 5 to $15 \%$ of live births in the world [4]. In this regard, one million and six hundred thousand births occur annually in Iran, of which 160,000 newborns are born 8 to 12 percent preterm, who require specialized care in neonatal intensive care units $[3,4]$.

Moreover, preterm babies' mothers face a premature and disabled baby who needs special equipment and care to survive. Subsequently, they experience a severe emotional shock after a difficult birth-giving process. As a result of this shock, they don't have enough capability and strength to take care of the baby [5] because dealing with a preterm baby or a patient admitted to the neonatal intensive care unit is one of the most sensitive and stressful stages in the life of parents, especially mothers. This confrontation, which considers as a major emotional crisis, can have adverse and long-term effects on mothers' lives [6].

The adverse effects of preterm birth on mothers are feelings of inability and intolerance, incompatibility, sadness, anxiety, fear, worry, guilt, anorexia, inability to breastfeed, depression, and sleep disorders [7, 8]. These mothers also experience significant changes in family relationships, work, social activities and family responsibilities, and even their parenting roles [9]. As they say, seeing a preterm baby inside an incubator or warmer under a ventilator or oxygen therapy, with multiple serums and lines attached to them, cause these mothers to doubt their ability to care for their babies. Many have become unable to adapt to the baby's condition and needs and subsequently have reported intolerance and intolerance in caring for their baby [8]. Therefore, mothers are less active in the pediatric section and they are not capable enough to participate in caring for their babies and playing their mother role [10].

Besides, one of the most fundamental roles and signs of attachment between mother and baby is breastfeeding [11]. Accordingly, the development of a sense of self-efficacy in breastfeeding in mothers of preterm infants improves their ability to care about their infants to reach them to a stable condition. In other words, breastfeeding self-efficacy means believing in one's ability to breastfeed a baby [12]. Breastfeeding self-efficacy is influenced by four essential factors, including past breastfeeding experiences, surrogate experiences (watching mothers who have been successful in breastfeeding), verbal encouragement by influential people in the mother's life (spouse, friends, family, and treatment team, especially nurses) and physical and mental illnesses (postpartum depression, anxiety, and fear) $[12,13]$.

Medical personnel, particularly nurses in the neonatal intensive care unit, can promote mother-infant interaction, attachment, and strengthen resilience and self-efficacy in breastfeeding. Furthermore, they can encourage and support them to stay in bed with their baby and engage in care procedures $[14,15]$. By advising and helping the mothers of these babies, nurses can promote a sense of self-efficacy in breastfeeding. Subsequently, mothers can experience a sense of empowerment, self-efficacy, and more peace in life [16]. 
According to what has been mentioned in previous studies, kangaroo care is one of the most influential participatory care in promoting the health of infants and improving mothers' sense of empowerment. Kangaroo care takes the form of skin-to-skin contact between mother and infant and concerns the three dimensions of infant health, mother-infant relationship, and maternal satisfaction and empowerment [15]. Concerning this, studies have examined the effect of kangaroo care on preterm infants and their mothers in recent years. Particularly, recent investigations have shown that kangaroo care enhances the physical health indicators of preterm infants $[11,17]$. However, kangaroo care is a pattern of two-way interaction between mother and baby, so in addition to infants, it can also affect mothers' physical and mental health indicators $[10,11,17]$. That's why Kangaroo care stimulates the secretion of oxytocin and decreases cortisol. Following these chemical happenings, the body can increase the mother's sense of calm, vitality, and pain tolerance, and reduces the mother's stress, anxiety, and worry [18]. Although there are many studies in these areas, few studies have examined the effects of kangaroo care on mothers of infants, especially mothers of preterm infants admitted to the neonatal intensive care unit $[7,16,19]$. In this regard, Macedo et al. (2007), Bigelow et al. (2012), and Faramarzi et al. (2013) have shown that kangaroo care calms, makes mothers feel better, empowers, and more tolerant, and can reinforce these feelings $[8,15,20]$. In addition, researchers such as Svensson et al. (2019), Yilmaz et al. (2019), and Zhang et al. (2019) have reported that kangaroo care increases mothers' confidence in caring for their babies [21-23]. These studies also revealed that mothers needed less help with breastfeeding and reported more breastfeeding self-efficacy [22]. Consequently, most of these investigations assessed the impact of kangaroo care on resilience, breastfeeding self-efficacy of infants using pamphlets and educational videos, and reported its benefits. Hence, it seems that examining the influence of kangaroo care by role-playing due to performance and role-playing before the actual performance can be practical and useful on the physical and mental health of mothers of preterm newborns. Wherefore, since a specific study has not evaluated the effect of kangaroo care by role-playing method on resilience and breastfeeding self-efficacy, we decided to study the influence of kangaroo care by role-playing method on resilience. Design and implement breastfeeding self-efficacy for mothers of infants admitted to the neonatal intensive care unit.

\section{Methods}

\section{Study design}

The present study was a randomized controlled trial with two parallel groups, one interventions and a control group conducted in a one-blind manner in one a hospital with two neonatal intensive care units affiliated to the University of Medical Sciences in western Iran from November 2020 to Murch2021.

\section{Ethical consideration}

The study design was approved by the Ethics Committee of the Hamadan University of Medical Sciences (Umsha.rec.1399.1042), and recorded at the Clinical Trials Center. Also, at the beginning of study, the researcher introduced herself and explained the aims of this study, and the written informed consent was 
obtained from all the participants after providing them with sufficient information on the study. The participants were assured that all information would remain confidential. The researcher created the opportunity for participants to inform the researcher about their withdrawal from the study at any stage of the study and assured them that their lack of participation or withdrawal would not have any consequences for them.

\section{Study population and Sample size}

Mothers of preterm infants admitted to two neonatal intensive care units participated in this study. Inclusion criteria included the following: Preterm infants weighing 1,800 grams or less. Have a minimum Apgar score of 5 minutes, 7 or higher at birth, 30 weeks and older at birth. These babies must be single, healthy, without severe physical disorders, and have not had surgery. It is worth noting that these babies were not undergoing phototherapy. Also, the baby should have the conditions to leave the incubator and warmer according to the opinion of a specialist doctor. The baby can suck on the mother's breast. The absence of psychiatric problems (depression, bipolar, etc.) in mothers, such as non-use of psychiatric drugs and tobacco by mothers was also considered. In addition, mothers were 18 years of age or older, and mothers were able to read and write. The second delivery was the first infant admitted to the intensive care unit and the mothers' willingness to participate in the study. Exclusion criteria also included the following: occurrence of unexpected physical problems for infants during the study, the unwillingness of mothers to continue the study, death, and transfer of the baby to another hospital, absence of mothers on one day of intervention (one day of mothers in The ward is not present and kangaroo care is not done).

The sample size in this study was selected according to the study conducted by Yilmaz et al. [22] With $\beta$ $=80 \%$ and $a=0.05$. The samples were estimated to be about 39 samples with a loss of $10 \%$ in each group and 78 mothers were randomly assigned to one of the two experimental and control groups. 3 infants from each group were excluded from the study due to changes in the physical condition and transfer to another hospital (Figure 1).

$$
n=\frac{\left(z_{1-\frac{\alpha}{\gamma}}+Z_{1-\beta}\right)^{\curlyvee}\left(\delta_{1}^{\curlyvee}+\delta_{\curlyvee}^{\curlyvee}\right)}{\left(\mu_{1}-\mu_{\curlyvee}\right)^{\curlyvee}}
$$

\section{Recruitment and allocation}

After determining the sample size, a total of 78 patients were screened for eligibility; then were enrolled and randomly assigned to one of two groups using block randomization with a volume of 2and an allocation ratio of 1:1 using a computer-generated randomization schedule, stratified by parity (two strata: first and second). this study was done a one-blind manner, so, questionnaires were collected by a researcher who was not aware of the assignment of individuals in the groups. 


\section{Intervention}

In the experimental group of mothers, each mother individually attended two 30 minutes long-sessions in the conference room of the hospital. These mothers were taught how to take care of their arms using baby modeling. To educate these mothers, the researcher first talked to the mothers in this group and answered their questions about how to care for, hug the baby, how to look at and caress the baby by the mother. He then helped them wear a special blouse designed according to the kangaroo care standard and protocol. These blouses were mostly loose, with sleeves, short buttons with a distance from the front of the garment that could be easily opened and closed, which were given to them by the researcher. The mother then sits in a comfortable chair in a semi-sitting position or in any position she feels comfortable following the next steps. The researcher placed a baby model with only a hat and diaper vertically between our mother's breasts to teach her kangaroo care. After this step, the researcher closes the bottom buttons of the mother's blouse. This model was completely placed in the mother's breast and in the fabric fence, and the possibility of falls and hypothermia was reduced. The researcher then stood next to the mother and asked her to try to make eye contact with the model while smiling and looking at her, caressing her, and also talking to her. Mothers in the intervention group, after training in embrace care by role-playing method, performed embrace care three times a day (once per shift) and each time for 30 minutes for 7 days with full supervision and accompaniment of the researcher. Mothers in the control group received a booklet training routine. Questionnaires completed by mothers before the intervention, one day after, and one week after discharge were collected by a researcher who was not aware of the assignment of individuals in the intervention and control groups.

\section{Outcomes and data collection}

\section{Connor and Davidson Resilience Scale}

The scale used in this study was a scale designed by Connor et al. (2003) in the United States to measure resilience. This scale has 25 questions in five areas (individual competence and competence 8 questions, tolerance of negative effects and strength against stress 7 questions, positive acceptance of change 5 questions, self-control 4 questions, and spiritual effects 5 questions). The questions on this scale are scored from zero (completely incorrect) to four (always correct) based on Likert scores. The maximum score in this questionnaire is 100 and the minimum score is zero, and a higher score indicates more resilience. The face validity, content, and also reliability of this questionnaire have been estimated by Ahangarzadeh Rezaei et al. (2015). This study mentioned that, the reliability of this instrument has been reported through Cronbach's alpha method about 0.82 [24].

\section{Breastfeeding self-efficacy questionnaire}

One of the important instruments in measuring breastfeeding self-efficacy in this study was the Dennis Lactation Self-Efficacy Self-Report Questionnaire, which was designed in 1999 by Dennis. This instrument can measure the confidence and capability of mothers breastfeeding based on 5-point Likert through 33 questions ( 1 strongly disagree to 5 strongly agree). All questions of this questionnaire are 
positive and the score range is 33-165. A higher score indicates more breastfeeding self-efficacy of mothers. The face validity, content, and also reliability of this questionnaire were performed by Dennis and its reliability was estimated at 0.96 by Cronbach's alpha method[25]. In Iran, Farhadieh et al. 1397 evaluated the face validity, content, and reliability of this questionnaire and reported it as an appropriate instrument for doing research [26].

\section{Data Analysis}

The collected data from the participants of the study were analyzed were by SPSS v.22 software through using descriptive statistics (mean-standard deviation, number-percent). After confirming the normal distribution of data by Smirnov Calligraphy method, analytical statistics with independent t-test and repeated measures analysis of variance were analyzed. It is worth mentioning that, an independent t-test was used to compare the mean scores of resilience and lactation self-efficacy independent groups in three times of measurement. Moreover, repeated measures analysis of variance was used to compare the mean of resilience and lactation self-efficacy between the two groups. As a result of this, the significance level was reported as 0.05 .

\section{Results}

The majority of the mothers of infants in this study had diploma and self- employed. Mean age of the mothers in the intervention group was $37.31 \pm 1.57$ years old, and mean age of the mothers in the control group was $37.42 \pm 1.41$ years old, and they had an income of about $\$ 120$ in each month in both groups.

The majority of the infants in these two groups were boys. Mean weight of the infants in the intervention group was $1721.42 \pm 1.92$ grams, and mean weight of the infants in the control group was $1756.21 \pm 1.74$ grams. Mean age of the infants in the intervention group was $31.42 \pm 1.52$ weeks, and mean age of the infants in the control group was $31.61 \pm 1.06$ weeks. There was no statistically significant difference in the distribution of demographic characteristics between the both groups $(P>.05)$. (Table1).

\section{Resilience in mothers of premature neonates}

Before the intervention, the both groups were not statistically significantly different in the resilience score $(P=0.71)$. Nonetheless, the repeated measures analysis of variance demonstrated a statistically significant difference in the score of the resilience in the each group one day and one week after performing the intervention $(P=<0.001)$. Moreover, the independent t-test revealed a statistically significant difference between the both groups in of resilience score one day and one week after performing the intervention $(P<0.01)$, So that the role playing method was more effective than the routine method (booklet training)( Table 2).

\section{Breastfeeding self-efficacy in mothers of premature neonates}

At the beginning of the study, the mothers in both groups performed poorly in breastfeeding self-efficacy, and there is not statistically significant difference in the breastfeeding self-efficacy scores at the 
beginning of the study between the both groups $(p=0.83)$, However, after the training kangaroo mother care, the mothers in the both groups made great progress in the breastfeeding self-efficacy scores. Intra group comparison of the breastfeeding self-efficacy scores during the study was statistically significant in the each group $(p=0.001)$, which showed that education kangaroo mother care by both methods (role playing and routine methods) were effective, but a statistically significant difference was observed between the both groups in one day and one week after performing the intervention $(p=0.01)$, So that the role playing method was more effective than the routine method (booklet training), (Table 3 ).

\section{Discussion}

The results of the present study have revealed that both role playing and routine methods are effective in promoting resilience and breastfeeding self-efficacy. But, the findings of this study showed that there was statistically significant differences among the two groups in improve the resilience and breastfeeding self-efficacy. It seems that role playing method is effective than routine method in promoting resilience and breastfeeding self-efficacy in mothers of premature infants in neonatal invasive care unite.

A study was not found that investigated the effect of kangaroo care training by roll modeling method on resilience and breastfeeding self-efficacy in mothers of premature neonates. Therefore, the effect of kangaroo care on resilience and breastfeeding self-efficacy was generally discussed.

Consistent with the findings of the present study, Ghazi et al. (2021) stated that home visit program based on the continuation of the mother and infant KMC had a positive effect on maternal resilience in mothers of premature infants, so that, there is a statistically significant difference in the mean resilience score of mothers one month after discharge between the intervention and control groups[27]. Although the results of this study show the effectiveness of KMC in improve of maternal resilience, but in this study mothers were trained KMC only the routine method (booklet training), and the experimental group continued the KMC after discharge, at home. Murty et al. (2018) express that KMC improve family resilience when they have low birth weight babies. KMC at home motivates family to manage problem effectively and at the end saving the baby from neonatal death[28]. This finding indicates that KMC is an effective method to improve the tolerance and resilience of families specially mothers with premature and low birth weight infants, which is in line with the findings of the present study. However, in this study also, mothers were trained KMC only the routine method (booklet training). It seems that if education is done in more objective and effective methods, such as role-playing, it can probably help families manage the crisis of premature birth and increase tolerance and resilience.

In addition, it is in line with present study, Rossman et al. (2017) state that training and the mother's ability to care for her infant in NICU has a positive effect on the maternal resilience, adaptability to the conditions[29]. Another study was done by Herizchi et al. (2018) also show that KMC has improved the adaptability and tolerance of mothers. Thus, the authors suggest kangaroo mother care should be taught continuously and with more efficient training methods to mothers of premature infants in order to promote maternal adaptation and improve the condition of newborn.[30] 
In this study also, breastfeeding self-efficacy in mothers of premature infants in neonatal invasive care unite improved in the role playing and routine groups, even role playing group had better performance in promote of breastfeeding self-efficacy than routine groups. Although, many studies have examined the breastfeeding self-efficacy in mothers of term infants, but few studies have examined the effect of KMC on the breastfeeding self-efficacy in mothers of premature infants in neonatal invasive care unite. In this regard, the study of Zhang et al. Showed mothers in the intervention group (KMC) reported more frequency and quality of breastfeeding than the control group. Therefore, it can be said that kangaroo care has significantly improved the number and quality of breastfeeding in mothers of premature infants [23]. Which is consistent with the present study, but in the present study, the mean score of breastfeeding self-efficacy was better in the control group (routine care with manual training) and the role-playing group A possible reason for this difference could be a difference in how is taught KMC. Consistent with the present study, Yilmaz et al. state that KMC is effective in promoting breastfeeding self-efficacy and they recommend that KMC to participate mothers in care of their infants and create a sense of empowerment in them and consequently help better development of infants [22]. Our study results are consistent with previous evidence from published literature that frequent skin-to skin contact between mother and infant is crucial to the successful transition to direct breastfeeding in preterm infants $[6,23,31]$ and initiation of exclusive breastfeeding in healthy full-term infants $[22,23]$

One of the notable limitations of the present study was the small sample size of the participants in the study. Therefore, it is recommended to conduct similar studies in the coming years in different communities, and with larger sample size. Moreover, in this study, mothers were followed for one week after intervention; it is suggested to follow up the effect of KMC on breastfeeding self-efficacy in mothers of premature infants for more weeks.

The results of the present study have revealed that both role playing and routine methods are effective in promoting resilience and breastfeeding self-efficacy. But, the findings of this study showed that there was statistically significant differences among the two groups in improve the resilience and breastfeeding self-efficacy. It seems that role playing method is effective than routine method in promoting resilience and breastfeeding self-efficacy in mothers of premature infants in neonatal invasive care unite.

A study was not found that investigated the effect of kangaroo care training by roll modeling method on resilience and breastfeeding self-efficacy in mothers of premature neonates. Therefore, the effect of kangaroo care on resilience and breastfeeding self-efficacy was generally discussed.

Consistent with the findings of the present study, Ghazi et al. (2021) stated that home visit program based on the continuation of the mother and infant KMC had a positive effect on maternal resilience in mothers of premature infants, so that, there is a statistically significant difference in the mean resilience score of mothers one month after discharge between the intervention and control groups[27]. Although the results of this study show the effectiveness of KMC in improve of maternal resilience, but in this study mothers were trained KMC only the routine method (booklet training), and the experimental group continued the KMC after discharge, at home. Murty et al. (2018) express that KMC improve family 
resilience when they have low birth weight babies. KMC at home motivates family to manage problem effectively and at the end saving the baby from neonatal death[28]. This finding indicates that KMC is an effective method to improve the tolerance and resilience of families specially mothers with premature and low birth weight infants, which is in line with the findings of the present study. However, in this study also, mothers were trained KMC only the routine method (booklet training). It seems that if education is done in more objective and effective methods, such as role-playing, it can probably help families manage the crisis of premature birth and increase tolerance and resilience.

In addition, it is in line with present study, Rossman et al. (2017) state that training and the mother's ability to care for her infant in NICU has a positive effect on the maternal resilience, adaptability to the conditions[29]. Another study was done by Herizchi et al. (2018) also show that KMC has improved the adaptability and tolerance of mothers. Thus, the authors suggest kangaroo mother care should be taught continuously and with more efficient training methods to mothers of premature infants in order to promote maternal adaptation and improve the condition of newborn.[30]

In this study also, breastfeeding self-efficacy in mothers of premature infants in neonatal invasive care unite improved in the role playing and routine groups, even role playing group had better performance in promote of breastfeeding self-efficacy than routine groups. Although, many studies have examined the breastfeeding self-efficacy in mothers of term infants, but few studies have examined the effect of KMC on the breastfeeding self-efficacy in mothers of premature infants in neonatal invasive care unite. In this regard, the study of Zhang et al. Showed mothers in the intervention group (KMC) reported more frequency and quality of breastfeeding than the control group. Therefore, it can be said that kangaroo care has significantly improved the number and quality of breastfeeding in mothers of premature infants [23]. Which is consistent with the present study, but in the present study, the mean score of breastfeeding self-efficacy was better in the control group (routine care with manual training) and the role-playing group A possible reason for this difference could be a difference in how is taught KMC. Consistent with the present study, Yilmaz et al. state that KMC is effective in promoting breastfeeding self-efficacy and they recommend that KMC to participate mothers in care of their infants and create a sense of empowerment in them and consequently help better development of infants [22]. Our study results are consistent with previous evidence from published literature that frequent skin-to skin contact between mother and infant is crucial to the successful transition to direct breastfeeding in preterm infants $[6,23,31]$ and initiation of exclusive breastfeeding in healthy full-term infants $[22,23]$

One of the notable limitations of the present study was the small sample size of the participants in the study. Therefore, it is recommended to conduct similar studies in the coming years in different communities, and with larger sample size. Moreover, in this study, mothers were followed for one week after intervention; it is suggested to follow up the effect of KMC on breastfeeding self-efficacy in mothers of premature infants for more weeks.

\section{Conclusion}


The results of this study showed that both role playing and routine methods are effective in promoting resilience and breastfeeding self-efficacy. Meanwhile, teaching kangaroo mother care through roleplaying method been more effective in promoting resilience and breastfeeding self-efficacy than routine methods in these mothers. So, we can recommend role playing and routine methods as therapeutic care methods in clinical settings at the beginning of the admission of infants in the neonatal intensive care unit to improve mother's resilience and breastfeeding self-efficacy.

\section{Abbreviations}

Kangaroo Mother Care (KMC), Neonatal Intensive Care Unit (NICU)

\section{Declarations}

\section{Ethics approval and consent to participate}

The study design was approved by the Ethics Committee of the Hamadan University of Medical Sciences (Umsha.rec.1399.1042), and recorded at the Clinical Trials Center. Also, at the beginning of study, the researcher introduced herself and explained the aims of this study, and the written informed consent was obtained from all the participants after providing them with sufficient information on the study. The participants were assured that all information would remain confidential. The researcher created the opportunity for participants to inform the researcher about their withdrawal from the study at any stage of the study and assured them that their lack of participation or withdrawal would not have any consequences for them.

\section{Consent for Publication}

Not applicable

\section{Availability of data and materials}

The datasets used and/or analyzed during the current study are available from the corresponding author on reasonable request

\section{Competing interests}

The authors declare that they have no competing interests.

\section{Funding}

This research did not receive any specific grant from funding agencies in the public, commercial, or not for profit sectors.

\section{Authors' contributions}


$\mathrm{FM}, \mathrm{KSH}, \mathrm{BN}, \mathrm{MB}, \mathrm{ES}, \mathrm{SKH}$ and SZM designed the study. They are responsible for data collection, SKH analyzed data. FM and MG drafted the primary manuscript, revised and approved the final manuscript.

\section{Acknowledgement}

This article is the result of a research project issued by Hamadan University of Medical Sciences. Hereby, we researchers send our sincerest thanks to respected authorities of Hamadan Midwifery and Nursing University, public hospitals in Hamadan, mothers, and other people who contributed us in carrying out this study.

\section{References}

1. Younge NE, Newgard CB, Cotten CM, Goldberg RN, Muehlbauer MJ, Bain JR, Stevens RD, O'Connell TM, Rawls JF, Seed PCJSr: Disrupted maturation of the microbiota and metabolome among extremely preterm infants with postnatal growth failure. Scientific Reports 2019, 9(1):1-12.

2. borimnejad I, ansari $k$, tatarpoor $p$, haghani h: Studying intra-hospital transportation situation of the high risk neonet in Neonatal Intensive Care Unit (NICU) Iranian Journal Of Critical Care Nursing (Ijccn) 2013, 6(3):197-204.

3. Ghojazadeh M, Hajebrahimi S, Pournaghi-Azar F, Mohseni M, Derakhshani N, Azami-Aghdash S: Effect of Kangaroo Mother Care on successful breastfeeding: A systematic review and Meta-Analysis of randomised controlled trials. Reviews on recent clinical trials 2019, 14(1):31-40.

4. Ravarian A, Vameghi R, Heidarzadeh M, Nariman S, Sagheb S, Nori F, Saeedershadi F, Norozi M: Factors Influencing the Attendance of Preterm Infants to Neonatal Follow up And Early Intervention Services Following Discharge from Neonatal Intensive Care Unit during First Year of Life in Iran. Iranian journal of child neurology 2018, 12(1):67.

5. Abul-Fadl AM, Khashaba AA, El Fiki UA, Saad NM: A Family-Centered Approach to Maternity care through Kangaroo Care and its Effect on Maternal Anxiety.

6. Aagaard H, Hall E: $\mathbf{5 0 0}$ Mothers' Experiences of Having a Preterm Infant in the Neonatal Care Unit: A Meta-Synthesis. Pediatric Research 2010, 68(1):256-256.

7. Athanasopoulou E, Fox JR: Effects of kangaroo mother care on maternal mood and interaction patterns between parents and their preterm, low birth weight infants: a systematic review. Infant mental health journal 2014, 35(3):245-262.

8. Bigelow A, Power M, MacLellan-Peters J, Alex M, McDonald C: Effect of mother/infant skin-to-skin contact on postpartum depressive symptoms and maternal physiological stress. Journal of Obstetric, Gynecologic Neonatal Nursing 2012, 41(3):369-382.

9. Izquierdo Renau M, Aldecoa-Bilbao V, Balcells Esponera C, del Rey Hurtado de Mendoza B, Iriondo Sanz M, Iglesias-Platas I: Applying Methods for Postnatal Growth Assessment in the Clinical Setting: Evaluation in a Longitudinal Cohort of Very Preterm Infants. Nutrients 2019, 11(11):2772. 
10. Karimi FZ, Bagheri S: The effect of kangaroo mother care immediately after delivery on mother-infant attachment 3 months after delivery. International Journal of Pediatrics 2016, 4(9):3561-3570.

11. Yilmaz F, Küçükoglu S, Özdemir AA, Ogul T, Aski NJTJoP, Nursing N: The effect of kangaroo mother care, provided in the early postpartum period, on the breastfeeding self-efficacy level of mothers and the perceived insufficient milk supply. 2020, 34(1):80-87.

12. Tork Zs, Karamollahi Z, Azgoli G, AKBARPUR BA, Sheikhan Z: Effect Of Support From The Mothers With Positive Breast Feeding Experience On Breast Feeding Pattern And Duration Among Primiparous Women Referred To Maternity Ward Of Ilam Hospital, 2010. Journal Of Ilam University Of Medical Sciences 2012, 20(2):9-16.

13. Parsa P, Boojar A, Roshanaei G, Bakht R: The Effect breastfeeding counseling on self-efficacy and continuation breastfeeding among primiparous mothers: a randomised clinical trial. Avicenna Journal of Nursing and Midwifery Care 2016, 24(2):98-104.

14. Adeli M, Alirezaei SJljon, research m: A comparative study of the effects of skin contact and kangaroo care on mother's anxiety. Pediatric Department, Benha Faculty of Medicine 2018, 23(4):327.

15. de Macedo EC, Cruvinel F, Lukasova K, D'Antino M, Famá E: The mood variation in mothers of preterm infants in Kangaroo mother care and conventional incubator care. Journal of Tropical Pediatrics 2007, 53(5):344-346.

16. Creti L, Libman E, Rizzo D, Fichten CS, Bailes S, Tran D-L, Zelkowitz PJSd: Sleep in the postpartum: characteristics of first-time, healthy mothers. Sleep Disorders 2017, 2017.

17. Hashemzadeh M: Effect of educational package on breastfeeding self-efficacy in postpartum period. Payesh 2014, 13(2):221-228.

18. Shobeiri F, Hajian S, Massoumi Z, Soltanian A: The Effect of Kangaroo Mother Care Counseling on Postpartum Blues, A Randomized Clinical Trial. Avicenna Journal of Nursing and Midwifery Care 2018, 26(2):113-119.

19. sevda S: The effect of aromatherapy using lavender on the quality of sleep in older people referred to monempour healthcare setting in Tabriz: A single-blindedrandomized clinical trial. Tabriz Tabriz University of Medical Sciences; 2017.

20. Faramarzi S, Badiee Z, Miri T, Khodadad Z: The effectiveness of kangaroo mother's care (KMC) method of low birth weight infants on maternal adjustment. Iranian Journal Of Nursing Research 2014, 8(4):29-41.

21. Widström AM, Brimdyr K, Svensson K, Cadwell K, Nissen E: Skin-to-skin contact the first hour after birth, underlying implications and clinical practice. Acta Paediatrica 2019, 108(7):1192-1204.

22. Yilmaz F, Küçükoglu S, Özdemir AA, Ogul T, Aski N: The effect of kangaroo mother care, provided in the early postpartum period, on the breastfeeding self-efficacy level of mothers and the perceived insufficient milk supply. The Journal of perinatal \& neonatal nursing 2020, 34(1):80-87.

23. Zhang B, Duan Z, Zhao Y, Williams S, Wall S, Huang L, Zhang X, Wu W, Yue J, Zhang L: Intermittent Kangaroo Mother Care and the Practice of Breastfeeding Late Preterm Infants: Results from Four 
Hospitals in Different Provinces of China. International Breastfeeding Journal 2020.

24. Ahangarzadeh RS, Rasoli M: Psychometric Properties Of The Persian Version Of "Conner-Davidson Resilience Scale" In Adolescents With Cancer. Journal Of Urmia Nursing And Midwifery Faculty 2015, 13(9):739-747.

25. Dennis CL: The breastfeeding self-efficacy scale: Psychometric assessment of the short form. Journal of Obstetric, Gynecologic, \& Neonatal Nursing 2003, 32(6):734-744.

26. Farhadieh A, Torkan B, Sadeghi N: Comparison of breastfeeding self-efficacy among mothers of hospitalized and non-hospitalized newborn, during the first week, the first month and the third month after childbirth. The J Urmia Nurs Midwifery Fac 2019, 16(12):914-926.

27. Ghazi M: The Effect of Home Visit Program Based on the Continued Kangaroo Mother Care on Maternal Resiliency and Development of Premature Infant: A Randomized Clinical Trial. International Journal of Community Based Nursing and Midwifery 2021, 9(1):64.

28. Murty Al: Saving Low Birth Weight Babies with Kangaroo Mother Care: Family Resilience and Social Capital as Success Factors. Journal of Modern Education Review 2018, 8:667-672.

29. Rossman B, Greene MM, Kratovil AL, Meier PP: Resilience in mothers of very-low-birth-weight infants hospitalized in the NICU. Journal of Obstetric, Gynecologic \& Neonatal Nursing 2017, 46(3):434-445.

30. Herizchi S, Hosseini MB, Ghoreishizadeh M: The impact of kangaroo-mother care on postpartum depression in mothers of premature infants. Int J Womens Health Reprod Sci 2017, 5(4):312-317.

31. Helmer CS, Thornberg UB, Frostell A, Örtenstrand A, Mörelius E: A Randomized Trial of Continuous Versus Intermittent Skin-to-Skin Contact After Premature Birth and the Effects on Mother-Infant Interaction. Advances in Neonatal Care 2020, 20(3):E48-E56.

\section{Tables}

Due to technical limitations, tables are only available as a download in the Supplemental Files section.

\section{Figures}




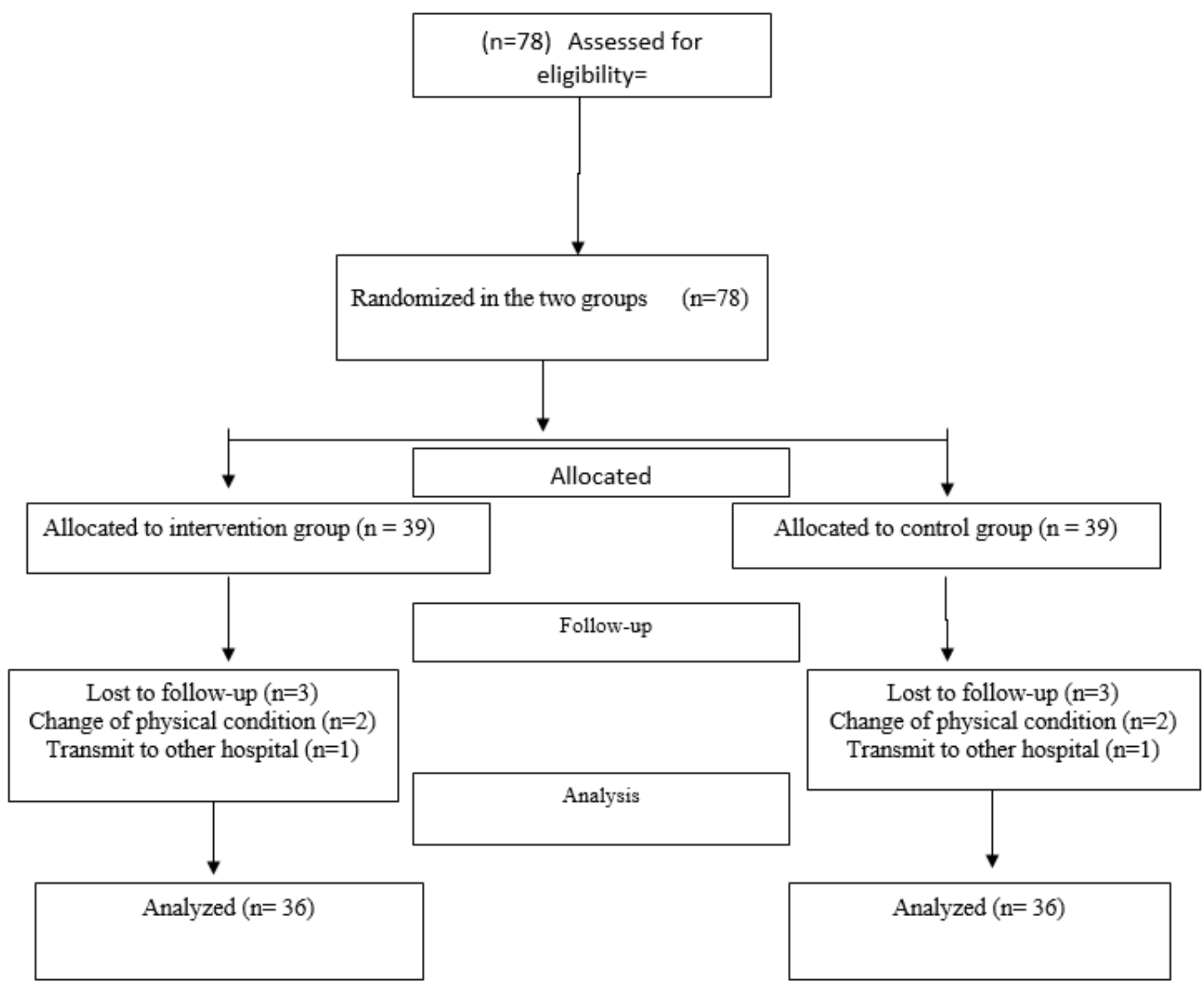

\section{Figure 1}

The Study Design

\section{Supplementary Files}

This is a list of supplementary files associated with this preprint. Click to download.

- Tables.docx 